\title{
Thin Films Under Chemical Stress
}

Detailed Summary of DE FG02 88ER13949

September 1, 1988 - April 1, 1991

The goal of work on this project has been to develop a set of experimental tools to allow investigators interested in transport, binding, and segregation phenomena in composite thin film structures to study these phenomena in situ. Work to-date has focused on combining novel spatially-directed optical excitation phenomena, e.g. waveguide eigenmodes in thin dielectric slabs, surface plasmon excitations at metal-dielectric interfaces, with standard spectroscopies to understand dynamic processes in thin films and at interfaces. There have been two main scientific thrusts in the work and an additional technical project. In one thrust we have sought to develop experimental tools which will allow us to understand the chemical and physical changes which take place when thin polymer films are placed under chemical stress. In principle this stress may occur because the film is being swelled by a penetrant entrained in solvent, because interfacial reactions are occurring at one or more boundaries within the film structure, or because some component of the film is responding to an external stimulus (e.g. $\mathrm{pH}$, temperature, electric field, or radiation). However all work to-date has focused on obtaining a clearer understanding penetrant transport phenomena. The other thrust has addressed the kinetics of adsorption of model $n$-alkanoic acids from organic solvents. Both of these thrusts are important within the context of our long-term goal of understanding the behavior of composite structures, composed of thin organic polymer films interspersed with Langmuir-Blodgett (LB) and self-assembled monolayers. Such structures might have unique functional properties, but the understanding of their structural chemistry is still at a primitive level. In addition there has been a good deal of work to develop the local technical capability to fabricate grating couplers for optical waveguide excitation. This work, which is subsidiary to the main scientific goals of the project, has been successfully completed and will be detailed as well.

\section{Transport Phenomena}

Rationale. Thin polymer films now pervade modern technology. In a number of their applications, it is important to understand how the film reacts to its environment. One technologically significant example is thin film swelling due to the diffusion of a solution component into the film; a well studied example being the differences in solubility between poly(methylmethacrylate) (PMMA) films which have been exposed to radiation (primarily electron or $\mathrm{X}$-ray) and those which have not been exposed. Since PMMA is a model positive resist material for the fabrication of microelectronic devices, its behavior has direct implications for the attainment of fine line lithographic featur, s, a hugely important component of our national technical capability. Understanding its behavior, however, requires a much clearer picture of the fundamental events in Case II transport. 


\section{DISCLAIMER}

This report was prepared as an account of work sponsored by an agency of the United States Government. Neither the United States Government nor anj agency thereof, nor any of their employees, makes any warranty, express or implied, or assumes any legal liability or responsibility for the accuracy, completeness, or usefulness of any information, apparatus, product, or process disclosed, or represents that its use would not infringe privately owned rights. Reference herein to any specific commercial product, process, or service by trade name, trademark, manufacturer, or otherwise does not necessarily constitute or imply its endorsement, recommendation, or favoring by the United States Government or any agency thereof. The views and opinions of authors expressed herein do not necessarily state or reflect those of the United States Government or any agency theieof. 
A number of researchers have recently examined transport phenomena in polymer films [19], and several classes of behavior have been identified and classified phenomenologically, based on the mechanism and the type of temporal dependence observed. Fickian, or Case I, diffusion is characterized by a $t^{1 / 2}$ time dependence in the front propagation and an error function concentration profile. This behavior is concordant with an interfacial polymer chain relaxation which is fast compared to the rate of movement of the solvent front. In contradistinction Case II diffusion is characterized by a linear (i.e. $t^{\mathfrak{l}}$ ) time dependence and a very sharp penetrant concentration profile. The different temporal dependence results because solvent front motion is faster than the polymer relaxation. Therefore, there is an important link between the phenomenologically observed temporal dependence and the molecular scale behavior, even though at present we cannot say very much about the molecular processes which drive the chain relaxation. Thus, if the tirne dependence of the diffusion process can be determined, the class of diffusion can be identified, and insight into molecular-scale behavior gained. Indeed characterization of the class of diffusive behavior operative in any given system is the important first step to understanding and controlling materials properties. Finally in some systems transport behavior follows neither the Fickian nor Case II limiting cases. These other types of temporal dependences are classified together as anomalous, or Case III, diffusion, indicating that the molecular mechanisms responsible for transport are more complex than in either of the simple models. It should be noted that these time dependences apply in the long-time limit, where the sensitivity of the solution to the diffusion equation to boundary and initial values is minimized.

It is important to determine the relative importance of these two diffusion mechanisms in thin polymer film model systems, because there is ample evidence to suggest that the diffusion process, even in a classic Case II example, will have a Fickian component on some distance scale. For example, calculations by Thomas and Windle for a $1 \mathrm{~mm}$ thick slab of PMMA being swollen with methanol (with diffusivity $D=10^{-10} \mathrm{~cm}^{2} / \mathrm{sec}$ and polymer viscous flow $\eta=5 \times 10^{-15} \mathrm{~m}^{2} \mathrm{MN}^{-}$ ${ }^{1} \mathrm{sec}^{-1}$ ) showed, ahead of the usual sharp diffusion front characteristic of Case II behavior, an exponential component of dimensions of the order of a few tens of $\mu \mathrm{m}$. Thus, for thin. films with total dimensions smaller than this Fickian diffusion component, it was an open question which type of behavior would dominate.

A number of different techniques have been applied to the analysis of diffusion in polymers. Among the methods utiliziu ivi depth profiling and diffusion analysis are NMR spectroscopy [10], ellipsometry [11-13], FTIR photoacoustic spectroscopy [14,15], secondary ion mass spectrometry (SIMS) [16], forward recoil spectrometry [17], and optical waveguide techniques [18-21]. Both SIMS and forward recoil spectrnmetry have the disadvantage of damaging or destroying the sample during the analysis and also cannot be performed in situ. NMR is not sufficiently sensitive for the analysis of thin films and is most appropriate under conditions where there is no concentration gradient. FTIR photoacoustic spectroscopy also requires fairly thick samples. Both optical waveguide techniques and ellipsometry are amenable to in situ characterization of transport in thin $(\leq 10 \mu \mathrm{m})$ films. In addition, both allow real-time data 
acquisition and are non-destructive. Optical waveguide techniques and ellipsometry approaches are reasonably comparable, however the waveguide experiments do have the advantage that molecularly specific information, since Raman scattering from the film constituents is used as the probe. The approach to characterizing the transport behavior in the current work involves the analysis of the changes in refractive index and thickness followed by optical modeling to determine the time dependence of the diffusion.

Eigenvalue Equation. All of the work carried out in this portion of the project focuses on the use of waveguide eigenmodes in thin polymer films as real-time experimental probes of film composition. This is possible, because the eigenmodes depend directly on the optical properties of the films through an eigenvalue equation,

$$
k_{0} n_{2} t_{2} \cos \theta_{2 j}+2 \tan ^{-1} \phi_{12}+2 \tan ^{-1} \phi_{23}=2 m \pi
$$

where $\theta_{2, j}$ is the internal waveguiding angle (in the ray-optics approximation), $t_{2}$ is the film thickness, $n_{2}$ is the film refractive index, and $\phi_{i j}$ is the phase shift angle upon reflection from the $i-j$ interface. In general the phase shifts also depend directly on the refractive indices of the two materials comprising the materials interface, thus all three terms in eqn. 1 depend directly on the optical properties of the film. Experimentally the waveguide angles (or equivalently the propagation constants) are measured, and the optical properties are then calculated by solving a coupled set of nonlinear transcendental equations. For three layers (i.e. a film and its two surrounding half-spaces) two eigenmodes are sufficient to determine the optical properties uniquely, and more than two eigenmodes will provide sufficient information to determine the precision of the measurement. If there are two thin films, then an expression similar to eqn. 1 results. In this case, however, at least four eigenmodes are required for an exact determination, while more are needed for an overdetermination. Of course, the calculations are more difficult in this case. However, satisfactory procedures for these four layer systems were worked out by our group several years ago [18-22]. The parameters determined are the refractive index and thickness for each layer, i.e. $\left\{n_{2}, t_{2}, n_{3}, t_{3}\right\}$. These may then be related to the overall composition of the layer by the effective medium relation,

$$
\frac{\varepsilon-1}{\varepsilon+2}=f_{1} \frac{\varepsilon_{1}-1}{\varepsilon_{1}+2}+f_{2} \frac{\varepsilon_{2}-1}{\varepsilon_{2}+2}
$$

where $f_{i}$ are the mole fractions of "ach of the constituents, $\varepsilon_{i}$ are the dielectric constants of each of the constituents, and $\varepsilon$ is the overall dielectric constant.

Optical waveguide phenomena have been studied using structures such as that shown in Figure 1. It is clear from previous studies that the distribution of mode angles changes with changes in the refractive index and thickness of the film; i.e. as the film index approaches the values of the superstrate and substrate refractive indices the region in which they are observed narrows, and the positions of the modes change. Also, the number of modes increases with 


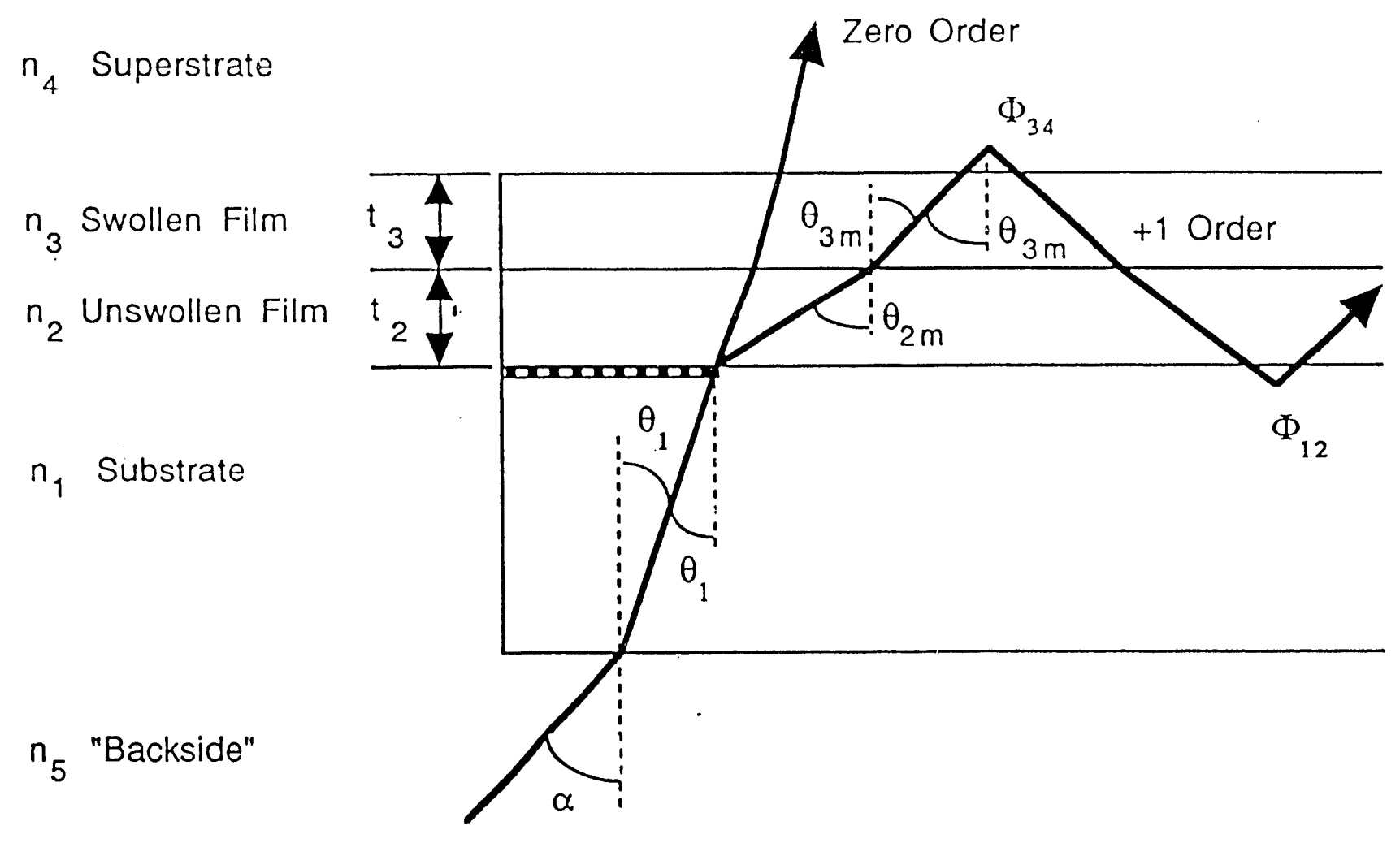

Figure 1: Sample Structure. A thin polymer film with unswollen refractive index $\mathrm{n}_{2}$ and thickness $t_{2}$ is deposited on a fused quartz substrate with refractive index $n_{1}$ and exposed to solvent, resulting in the two layer structure with the swollen film refractive index $n_{3}$ and thickness $t_{3}$. The lower layer, the unswollen film, decreases in thickness with time, while the upper layer, the swollen film, increases in thickness, both from front propagation and swelling. The environment around the sample is described by the superstrate refractive index, $n_{4}$, and the backside refractive index, $n_{5}$. $\alpha$ is the incident (observed) angle for the light, $\theta_{1}$ is the angle inside the substrate, $\theta_{2 \mathrm{~m}}$ is the guiding angle for mode number $\mathrm{m}$ in the unswollen film and $\theta_{3 \mathrm{~m}}$ is the angle in the upper layer of the film for mode m. $\Phi_{12}$ and $\Phi_{34}$ represent the phase shifts at the substrate-film interface and the film-superstrate interfaces, respectively. The +1 order diffraction of the grating is coupled into the film, while the zero order beam passes through the film. In order for guiding to occur $n_{2}>n_{3}>n_{1} \geq n_{4}, n_{5}$. 
increasing film thickness. In short, the measured propagation constants may be inverted to yield optical property data for the thin film(s) in the structure. First, a set of calculations, assuming a single homogeneous layer, is performed to obtain the general behavior of the structure and the amount of time required to reach equilibrium. This allows the more computationally intensive twofilm calculation to be performed only during the appropriate time regime. In order to solve the two-film problem, four unknowns must be determined, $n_{2}, t_{2}, n_{3}$, and $t_{3}$. We test for a Case II diffusion mechanism by assuming that we have two layers characterized by different optical properties, i.e. the swollen and unswollen film regions. The film system can be modeled in this manner, for Case II diffusion only, because the sharp concentration front divides the system into two regions with distinct chemical compositions (and thus optical properties), and because the boundary region is small (1-2\% of the total film thickness) compared to the overall dimensions of the film. In the analysis, the refractive indices of the two layers are known, and the eigenmode propagation constant data is inverted to obtain $t_{2}$ and $t_{3}$. The unswollen film refractive index, $\mathrm{n}_{2}$, is determined from scans collected with an air superstrate, while the swollen film refractive index, $\mathrm{n}_{3}$, is determined from scans collected after the film has come to equilibrium with the solvent, i.e. when the diffusion front has propagated through the entire film. With this information in hand the two thicknesses are determined by calculating an error surface,

$$
g\left(t_{2}, t_{3}\right)=\sum_{\text {modes }}\left|\beta_{j}^{\text {obs }}-\beta_{j}^{c a l c}\left(t_{2}, t_{3}\right)\right|^{2}
$$

where $\beta$ is the propagation constant for a given mode, and the squared differences are summed over all observed modes. Searching this error surface for the global minimum then yields the most appropriate values of $t_{2}$ and $t_{3}$. A pictorial description of this approach is given in Figure 2.

Model Systems: Poly(styrene). In our work we have concentrated on exploiting this experimental protocol in order to detail the dynamics of the swelling process in the model systems poly(styrene)/n-hexane and poly(2-vinylpyridine)/water. Poly(styrene) was chosen for study, because there has been a great deal of work in understanding transport of organic solvents in thicker films, because there is a great deal of experience working with thin films of the material in our laboratory, and because it presents a stringent test of the use of optical waveguides to study transport phenomena. Preliminary experiments showed that, as expected, there was no significant change in refractive index or thickness of the film, even after extended exposure tp eother water or methanol. In the remainder of the experiments $n$-alkanesolvents were used. The diffusion of $n$ alkanes in poly(styrene) films ranging from $50 \mu \mathrm{m}$ to $1 \mathrm{~mm}$ thick has been examined by several groups $[9,26]$. In all cases a linear time dependence was observed, indicating Cáse II diffusion, but neither the induction time nor exponential leading edge characteristic signatures of Case II transport have been observed. Also, the development of crazes, or micro-cracks, in the polymer were observed at the diffusion front and were attributed to the stress built up at the sharply-defined diffusion front. The crazes appeared during the front propagation, but were observed to heal in the swollen polymer region. As with other defects in the film the crazes can act as physical light scattering centers. 


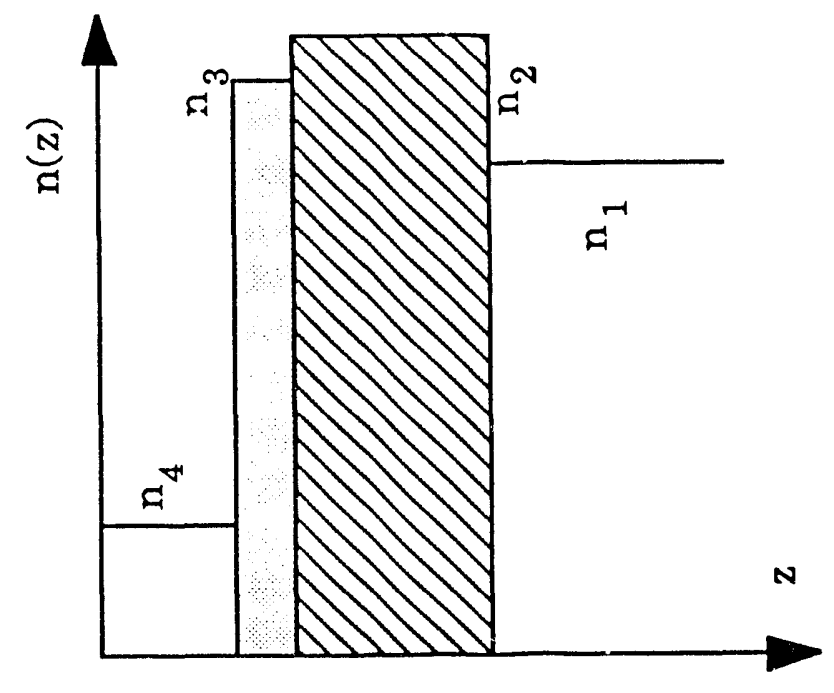

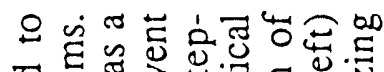

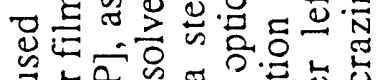

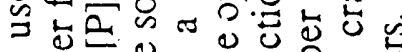

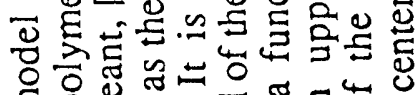

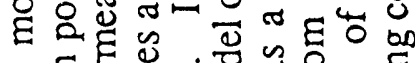

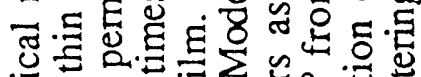

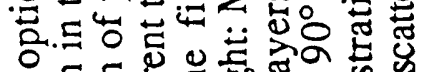

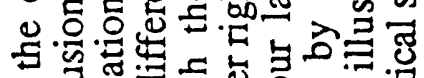

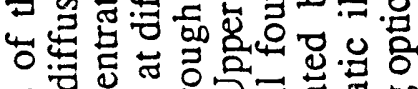

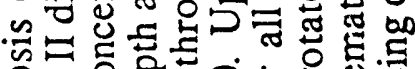

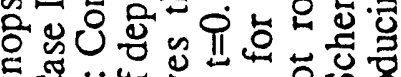

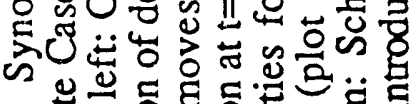

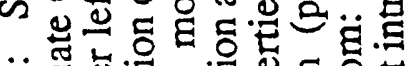

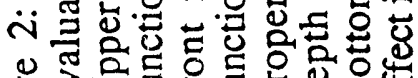

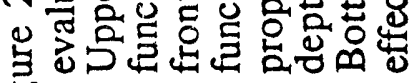
i.
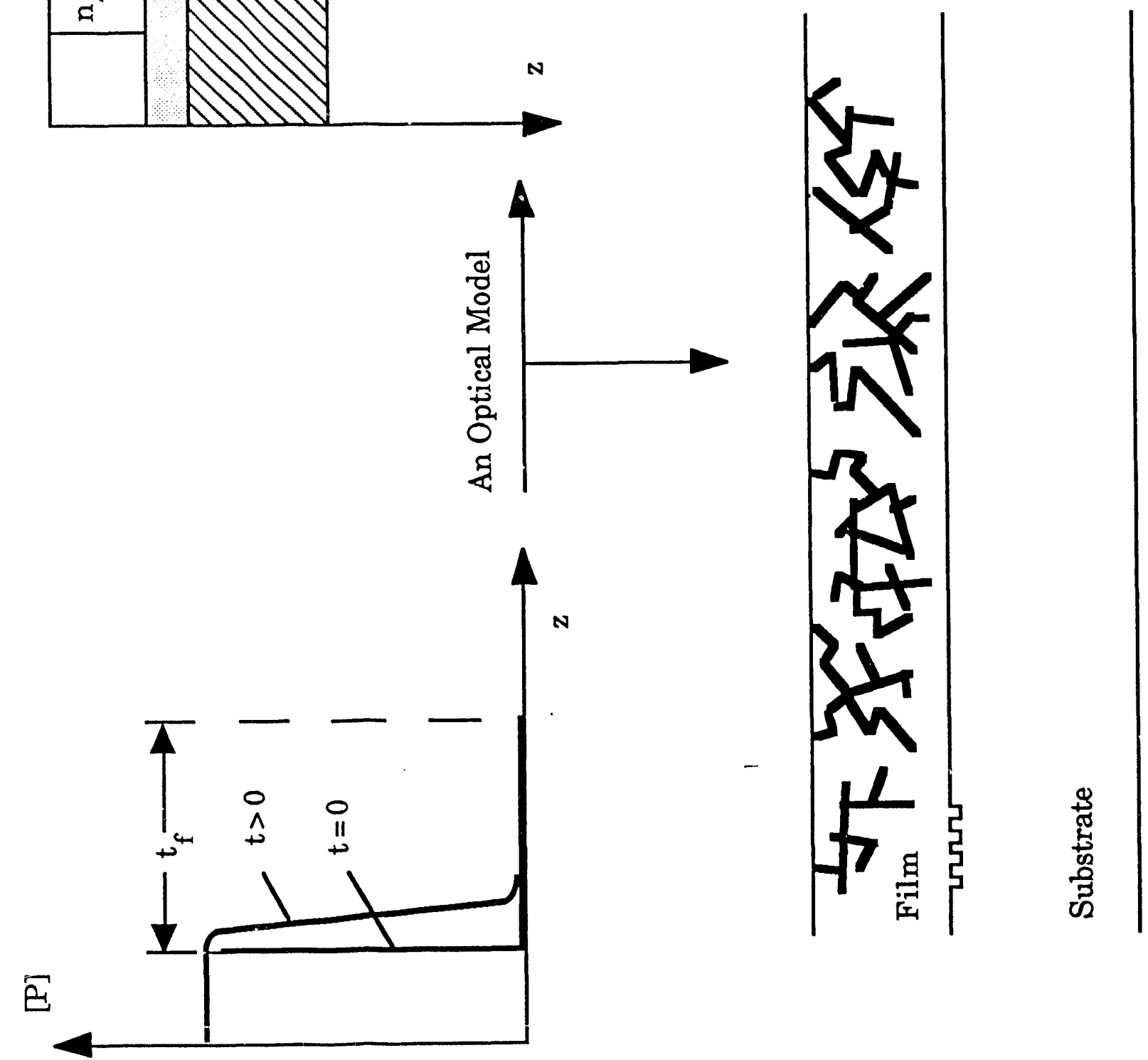
This physical scattering is important for the present experiments, because it introduces optical losses and reduces the intensity of the Raman scattering from the film. Thus, the number of modes which can be observed at any time during the evolution of the solvent front changes, becoming smaller as the extent of craze formation increases. However after stress relaxation the crazes are observed to anneal and the full eigenmode spectrum can once more be observed. A typical set of eigenmode distributions at different times for an $n$-hexane/methylcyclopentane mixture permeation is shown in Figure 3. Clearly for the least efficiently excited modes, i.e. those at the high and low end of the mode spectrum, less light is available for excitation of Raman scattering, which was being monitored. The crazing is instigated by the diffusion front, and when it reaches the substrate, the polymer relaxation, which has been occurring behind the front, anneals the crazes. The complete mode structure is again visible and givivs in intensity. While the crazing reaches its maximum at the point at which the diffusion front has just reached the substrate, the attenuation has become severe enough several hours before this point to make the modes undetectable. This behavior has been previously observed in macroscopic films [26], although the effect is much less ciramatic than that observed here.

The air data were then analyzed to obtain $n_{2}$ and the equilibrium scans after 9 hours were used to obtain $n_{3}$ using a one-film approximation. It should be emphasized that the one-film calculations performed after introduction of solvent, but before equilibrium has been attained, are clearly inappropriate. However, the weighted average values, which are obtained for the dielectric constant and thickness, serve as a useful indicator of global changes in film structure. Figure 4 exhibits the composite refractive index, obtained from the one-film calculations and the correct total thickness $\left(t_{2}+t_{3}\right)$, from the two-film calculations. The recovered refractive index is expected to decrease as the total sorbed solvent increases, since the solvent has a lower refractive index than the film, and indeed this is the observed behavior. The total thickness qualitatively matches the increase and subsequent decrease in film thickness predicted by Thomas and Windle [1-3]. Since this behavior is a characteristic feature of the Case II mechanism, this supports the dominance of Case II diffusion in this system. It should also be emphasized that this is the first experimental verification, in mm-scale films, of the shrinking after swelling behsvior and that these observations cannot be made by experiments sensitive to mass loading in the film.

While this behavior is distinctly characteristic of Case II diffusion, examining the front motion is more informative. This is accomplished by looking at the difference, $d_{2}(0)-d_{2}($.$) ,$ between the initial thickness (from the air scan), $d_{2}(0)$, and the values of the lower layer thickness, $d_{2}(i)$, and plotting this difference against time in order to determine the temporal dependence of the diffusion process. $\log -\log$ plots of this difference vs. time show two clear regions. From 0 to 1.25 hours the slope is 0.563 , while after 2.25 hours the slope is 1.099 . The initial region could be the result of the induction time associated with Case II diffusion. Independent experiments, with fresh films, on the individual solvent components showed a similar behavior for both components, confirming this explanation. The interpretation of the nonlinear time dependence of the early time regime was obtained by forcing the data into a Case II model. Thus, all that can be said about the early time behavior is that the front velocity is not linear in time. Of course, this is 


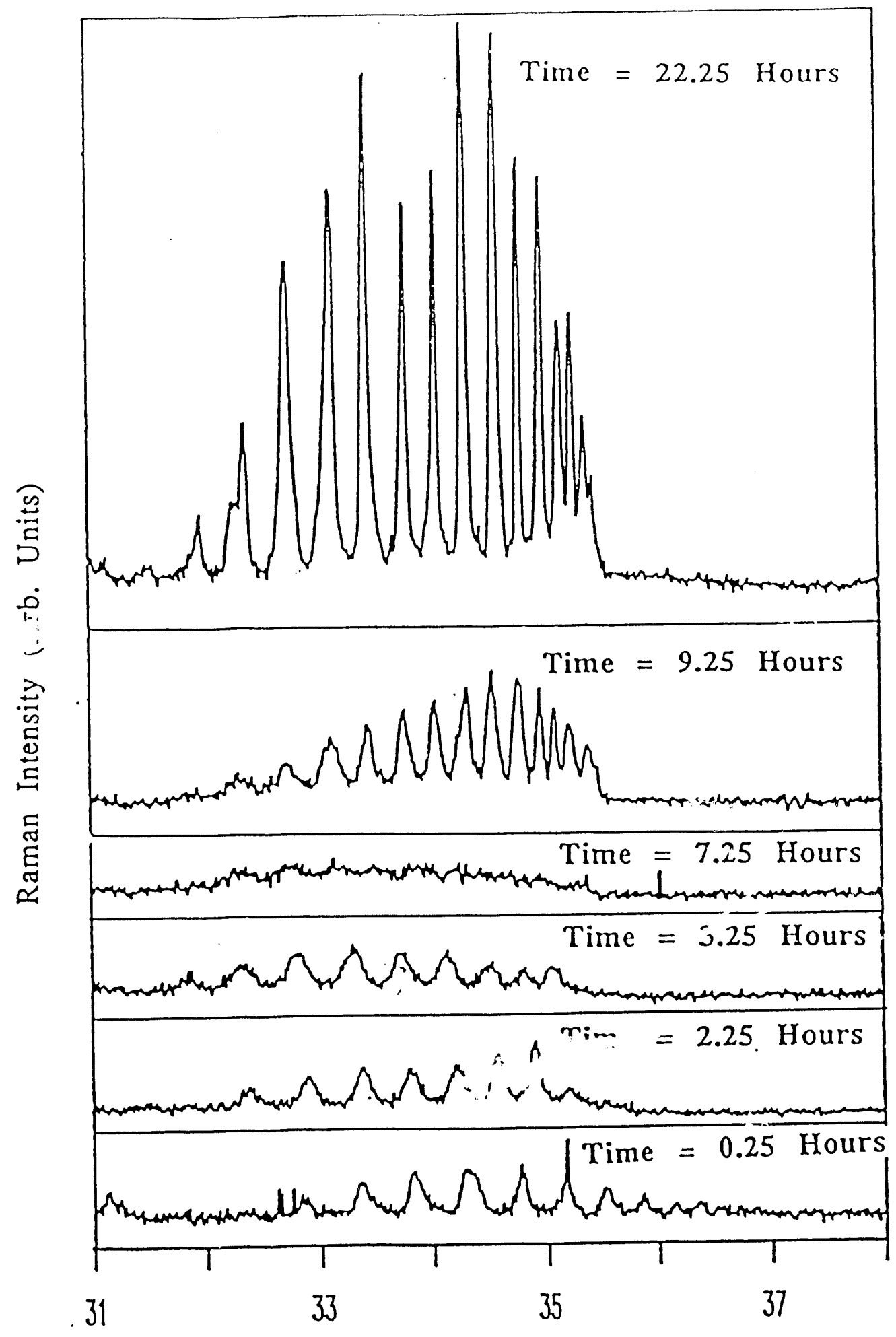

Angle (Degrees)

Figure 3: Representative angle scans of polystyrene in $\mathrm{n}$-hexane/methylcyclopentane mixture. The decrease in the mode intensity and the complete attenuation of the lowest and highest order modes until 9.25 hours, when they reappear and strengthen, was attributed to the crazing of the film which causes physical scattering of the guided radiation. 


\section{Total Thickness (Microns)}

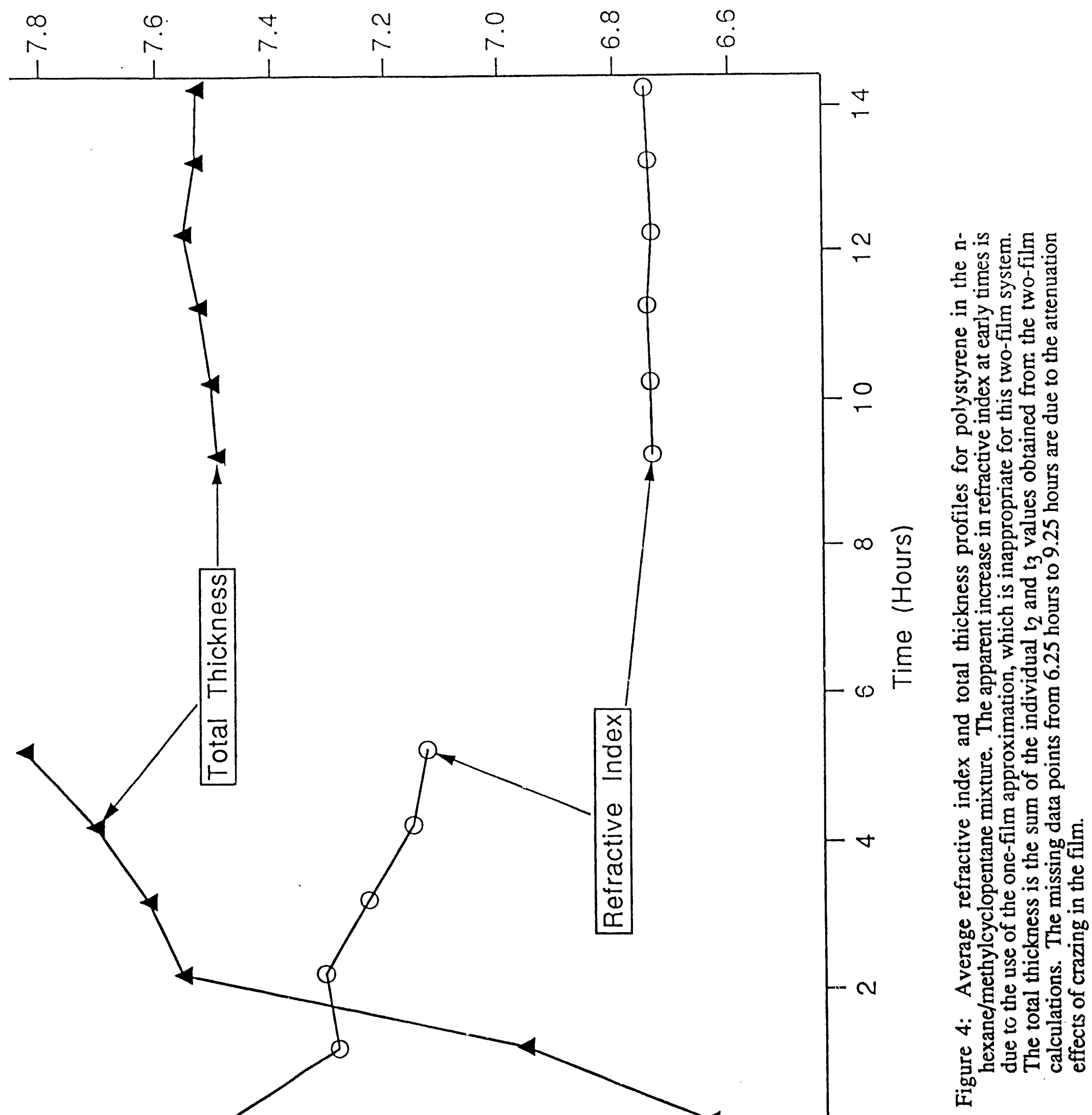


consistent with the induction time present in the Case II model [7]. The analysis of pure n-hexane diffusion in a thinner, $2.5 \mu \mathrm{m}$, polystyrene film exhibited behavior consistent with that of the mixture. When the front propagation was examined by plotting $\log \left\{d_{2}(0)-d_{2}(i)\right\}$ as a function of time, two regions were observed. Regression on all of the points, resulted in a slope of 0.712 , while removing the early time points (before $t=15 \mathrm{~min}$.) yielded a slope of 0.976 , in excellent agreement with the Case II linear time characteristic. Again the eariy data can be explained as the result of the induction time caused by the Fickian leading edge of the diffusion front.

In order to compare the results obtained here with the literature, it is necessary to compare diffusion front velocities. This is necessary because the traditional diffusion coefficient is not applicable to a Case II diffusion process, since it is based on a $t^{1 / 2}$ time dependence. The front velocity in n-hexane was $7.57 \times 10^{-8} \mathrm{~cm} / \mathrm{sec}$. Nicolais and coworkers [9] have reported a front velocity of $7.5 \times 10^{-7} \mathrm{~cm} / \mathrm{sec}$, and Sarti [4] has reported a value of $8.9 \times 10^{-7} \mathrm{~cm} / \mathrm{sec}$ at $35^{\circ} \mathrm{C}$ for $\mathrm{n}$ hexane diffusion in polystyrene. The apparent discrepancy with our data is easily explained by the difference in temperature, since our experiments were performed at ca. $25^{\circ} \mathrm{C}$, and possibly to differences in the density of film defects, such as cracks and pinholes, in the macroscopic films, which will accelerate the apparent front velocity. Sarti also reports the values at several higher temperatures and the data obtained in this study at $25^{\circ} \mathrm{C}$ for $\mathrm{n}$-hexane diffusion into polystyrene fits an extrapolation of Sarti's data.

Model Systems: Poly(2-vinylpyridine). There is a strong interest in our laboratory in examining thin polymer film systems which are compatible with bioprocess streams and other biologically relevant sample media. Thus we began a search for a material with app. spriate optical properties which could be swollen, but not dissolved, by aqueous solutions. Extensive experiments with poly(styrene sulfonate) under various cross-linking conditions failed to yield films which would swell without dissolving. We tried a wide variety of different cross-linking reaction conditions, and while we were able to affect the time it took to dissolve films a few $\mu \mathrm{m}$ thick, we were not able to achieve a sufficient cross-link density to render the material insoluble. Thus we turneci to different materials and found that poly(2-vinylpyridine) (P2VP) provided excellent aqueous swelling characteristics. P2VP was quickly swollen by water. Equilibrium film composition was obtained within the first five minutes after immersion (this is the time that it takes to relocate the eigenmodes in angle space and take an angle scan), and the same eigenmode distribution was obtained many hours later. Composi ional changes are illustrated in Figure 5. When removed from the aqueous environment and dried, the film recovered its original optical properties, i.e. $\{n, t\}_{\text {dry }}=\{1.6050,3.27 \mu \mathrm{m}\} \rightarrow\{n, t\}_{\text {swollen }}=\{1.5247,4.03 \mu \mathrm{m}\} \rightarrow\{n, t\}_{\text {dry }}=$ $\{1.6073,3.29 \mu \mathrm{m}\}$. Cycling experiments have shown that these P2VP films are quite durable. Thus all further developmeni work on aqueous environments has been performed with P2VP as the base material.

At this point the most interesting feature of transport in P2VP films is the large disparity in rates between solvent $\left(\mathrm{H}_{2} \mathrm{O}\right)$ and our model permeant, fluorescein isothiocyanate (FITC). As indicated above equilibrium swollen film properties were obtained in the first five minutes for a 

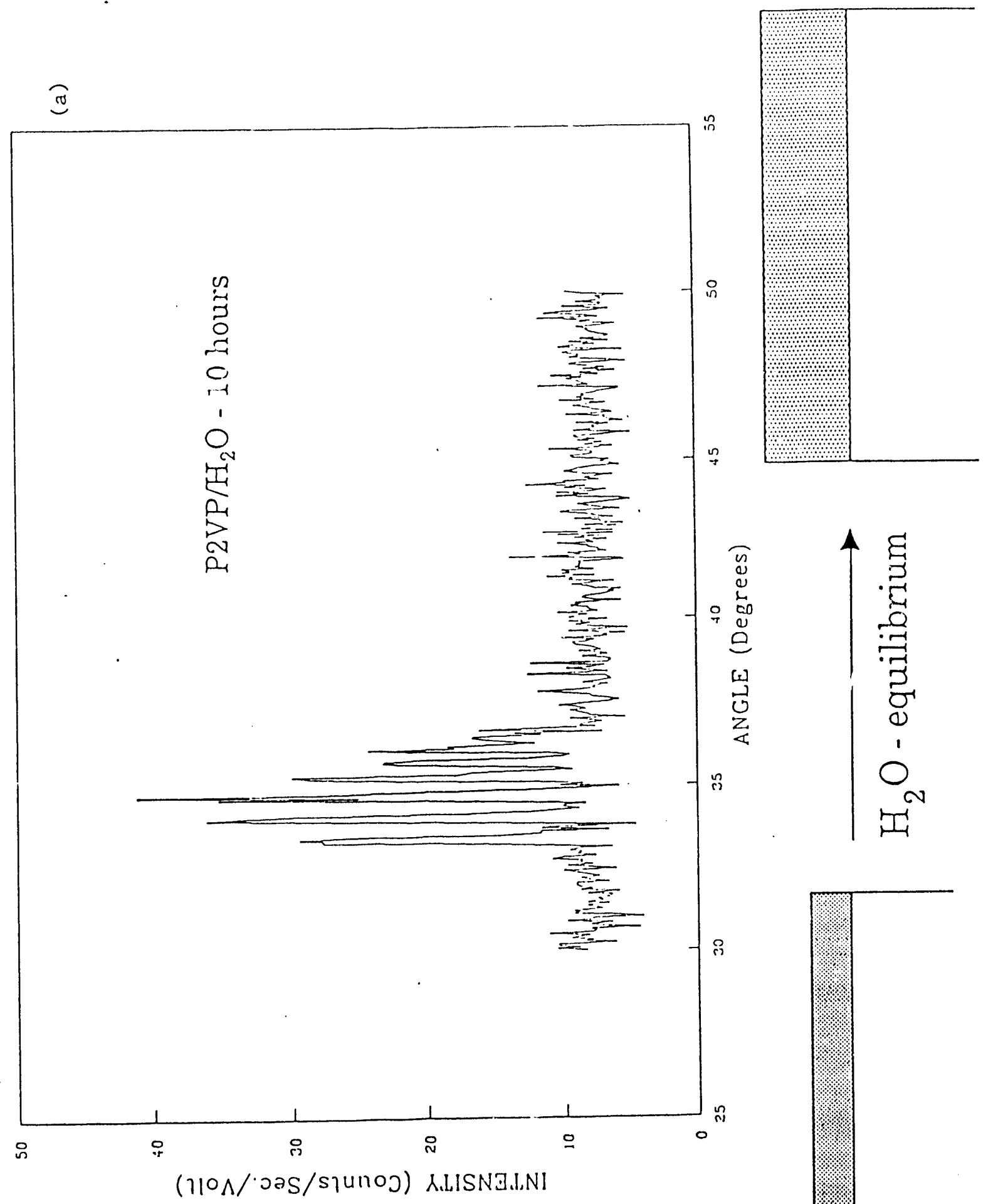

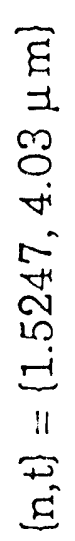

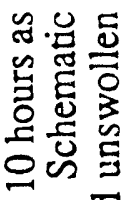

+

ㄴ.

흘

․

11 . 응.5

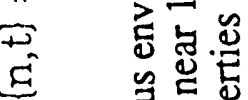

웡

잉융

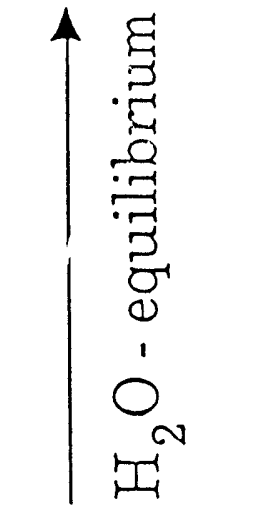

ह

도윰

.

톨 옹

007

政

ธ $0 . \Xi$

药

홍 ․ํㅇ

द .0

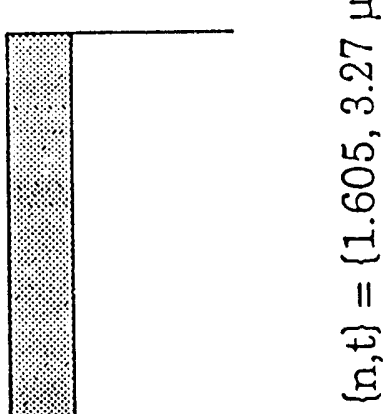

$\exists \quad$.

웡

r 宅的

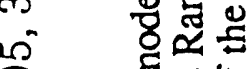

잉녕

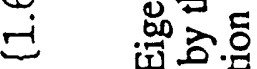

I ब

它总总完 
typicai film. Since this is too fast to follow directly by the techniques discussed above, we can only choose a model for the diffusion and use that model for comparison. Picking the Fickian model, which seems more reasonable in light of the very fast swelling behavior, we set a lower limit on the diffusion coefficient of water in F2VP at D $\geq 1.5 \times 10^{-10} \mathrm{~cm}^{2} / \mathrm{sec}$, while the apparent diffusion coefficient for FITC is much smaller.

In order to extract the diffusion coefficient for FITC we use the P2VP film as an overlayer on a much thicker optical guiding layer. In this situation the waveguide must be modeled to include the effects of (1) radiation loss in the x-direction (i.e. guiding direction) from FITC absorption, and (2) increasing concentrations of FITC in the film at all $x$-positions as a function of time as it diffuses into the film. Tinis is solved by irvoking the following model. First, we assume Fickian diffusion of FITC into the film (z-direction) giving a time-dependent FITC concentration, $F(t)$,

$$
F(t)=\int_{0}^{W} \frac{1}{2} F(\text { bulk }) \operatorname{erf}\left(\frac{z}{2 \sqrt{D x}}\right) d z
$$

which has the solution,

$$
F(t)=\frac{F(\text { bulk })}{2 \sqrt{\pi}}\left(-w \sqrt{\pi} \operatorname{erfc}\left(\frac{w}{2 \sqrt{D t}}\right)+2 \sqrt{D t} e^{-w 2 / 4 D t}\right)+\left(\frac{F(\text { bulk }) \sqrt{D t}}{\sqrt{\pi}}\right)
$$

The measured quantity is the fluorescent intensity as a function of time after introduction of FITC into the cell, which is given by,

$$
I(t)=\int_{0}^{I} F(t) I_{0} e^{-\varepsilon F(t) x d x}
$$

which has the solution,

$$
I(t)=I(0)(1-e-\varepsilon 1 F(t))
$$

where $\varepsilon$ is the molar absorptivity in the film, and $l$ is the length over which radiation is collected. We then fit this model to the experimental fluorescence intensity data. Results from fits on three separate samples are siown in Figure 6. Clearly very consistent results are obtained for the Fickian diffusion coefficient and the molar absorptivity. The parameter $\mathrm{I}(0)$ is an experimental scaling parameter which takes into account, among other things, the coupling efficiency and the film thickness. Thus, it is expected to vary from sample-to-sample. 

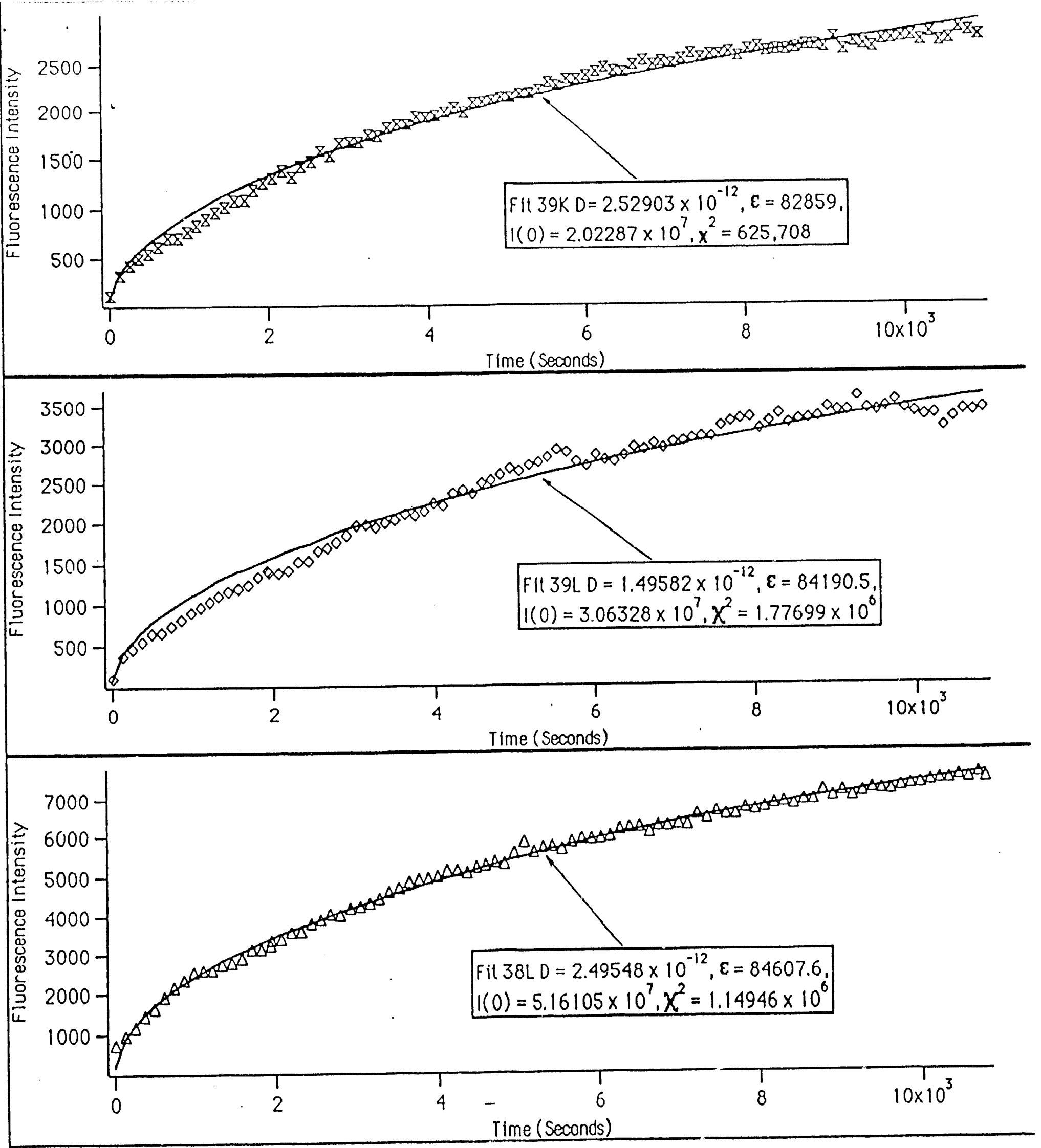

Figure 6: Plots of the FITC fluorescence intensity as a function of time of permeation into a water- .. swollen P2VP film. Lines are fits of eqn. 7 to the data. The three separate plots were obtained on different samples on different days, illustrating the axcellent reproducibility of the swelling and diffusion behavior. 
A final interesting observation about this experimental system is that the FITC in the film is stable to leaching under pure water conditions. This observations argues for a specific binding of the FITC to a portion of the polymer matrix. One possibility is the electrostatic binding of the negatively charged carboxylate group to a protonated pyridinium moiety on the polymer. We are currently investigating this possibility by performing the leaching experiments at varying ionic strength. Whatever the nature of the resistance to leaching, it is interesting to note that the implications the observation has for the microscopic transport processes which give rise to a Fickian-like macroscopic behavior in this system. One possibility is that the kinetics of binding are fast and irreversible. Thus, initially FITC molecules are bound to the outer layers of the film. Subsequent molecules might have to diffuse past this bound-FITC layer before reaching open binding sites with which to react. This is our current working hypothesis, and we are taking two approaches to investigating it. First we are building a model which will emulate the macroscopic behavior of this microscopic behavior. Second, we are preparing to perform optical depth profiling $[21,22]$ experiments in grating-coupled P2VP waveguides. Previously such experiments would not have been possible due to the strong tendency to photodegrade the photolabile FITC probe molecule, however significant improvements in our excitation and collection schemes now permit data to be collected with negligible degradation.

In conclusion, these experiments have clearly demonstrated the utility of optical wayeguide techniques for the study of diffusion in thin ( $\mu \mathrm{m}$-scale) films. These techniques are unique in their ability to study diffusion in real time for films in this size regime. In addition, the observation of the Fickian leading edge and induction time effects directly add to the experimental distinction between Case I and Case II diffusion mechanisms. The ability to distinguish between diffusion mechanisms on the basis of the time dependence of the front propagation and front velocities are also straightforward in the optical waveguide technique and agree with the predictions found in the literature for the systems studied here. The single largest drawback to the technique presented here is the length of time required to analyze the data. However, since the analysis can be done off-line, the time constraint is not too severe and will become even more facile with the advent of even more powerful computational resources. Thus, we expect that integrated optical techniques will come to play an important role in the study of transport phenomeis in thin organic films.

\section{Adsorption Phenomena}

Rationale. A key goal in our laboratory is to develop methods for studying composite structures, in which different structural elements are brought together to implement different functions. One type of composite structure is that cumposed of polymer layers interspersed with ultrathin (one to, at most, a few molecular layers) am such a structure presupposes a detailed understanding of both types of structural units. The foregoing portion of this progress report details our efforts to develop experimental tools to examine thin polymer layers. A parallel set of experiments designed to understand the assembly and structural features of amphiphilic layers has focused on the adsorption kinetics of $n$-alkanoic acids on Ag surfaces from cyclohexane solution. These processes have been followed both by 
observing shifts in the surface plasmon resonance condition and by temporally-resolved in situ surface Raman scattering.

The traditional means of forming an organic monolayer is to transfer a mechanically compressed, densely packed, oriented monolayer to a solid substrate by the Langmuir-Blodgett (LB) technique. LB monolayers have certain drawbacks, however. First, because a LB monolayer is not chemically bonded to the surface, it can often be removed by simple rinsing with a solvent. Second, such layers are typically only metastable and over time can relax to more stable structural forms with different physical properties. Another way to prepare a stable, densely packed, oriented film is through self-assembly. The chemisorbed films thus formed are mechanically robust and thermodynamically stable [27]. In the vast majority of studies on selfassembled films the emphasis has been placed on the properties of the film formed and not on the processes involved in making the film. While equilibrium studies do address the questions of heats of formation, surface density, wetting characteristics, surface disorder and others, they remain mute on the kinetic details, intermolecular interactions, van der Waals interactions, and the activation energy of the adsorption process. Insights gained from proper modeling of the adsurption process can be useful in understanding the role the solvent and functional groups play in the adsorption process and how modification of the materials can alter the structure of the films, by altering the manner in which the film is formed.

Methodology. In this set of experiments we have been following both the fast (secondsminutes) and slow (days) kinetics of $n$-alkanoic acids displacing a preadsorbed layer of acetic acid at a freshly prepared $\mathrm{Ag}$ surface from cyclohexane, i.e.

$$
\mathrm{CH}_{3}\left(\mathrm{CH}_{2}\right)_{n} \mathrm{CO}_{2} \mathrm{H}_{\text {soln }}+\mathrm{CH}_{3} \mathrm{CO}_{2} \mathrm{H}_{\text {surf }} \rightarrow \mathrm{CH}_{3}\left(\mathrm{CH}_{2}\right)_{n} \mathrm{CO}_{2} \mathrm{H}_{\text {surf }}+\mathrm{CH}_{3} \mathrm{CO}_{2} \mathrm{H}_{\text {soln }}
$$

Our first concern was in developing a technique to determine submonolayer coverages with high precision over extended time periods on low surface area substrates. To meet these objectives we exploited the adsorption induced shifts in the surface plasmon dispersion curve at the Ag-solution interface. Adsorption of molecules at the interface between a thin metal film and the bulk solution shifts the position of the surface plasmon resonance. Since the detailed shape of the reflectivity can be calculated directly from a simple optical model, fits of experimental plasmon resonance curves can be used to characterize sub-monolayer changes in the composition of a surface film with high precision. A schematic diagram of the coupling apparatus and some typical data are shown in Figure 7.

We used a non-traditional method of procuring surface plasmon data, however. Typically a well-collimated beam is brought into a prism, and different values of $\mathrm{k}_{\mathbf{x}}$ are accessed by changing the angle of excitation. To eliminate the need to scan the prism, Matsubara, et.al. [28] developed a Fourier optics approach which brings a cone of light into the prism to access a range of photon wavevectors. The removal of moving parts means faster acquisition times, typically $10 \mathrm{msec}$ in our system, meaning kinetic studies can be carried out on the millisecond time scale. We also insert a half-wave plate prior to the focusing lens to allow excitation of either transverse electric 


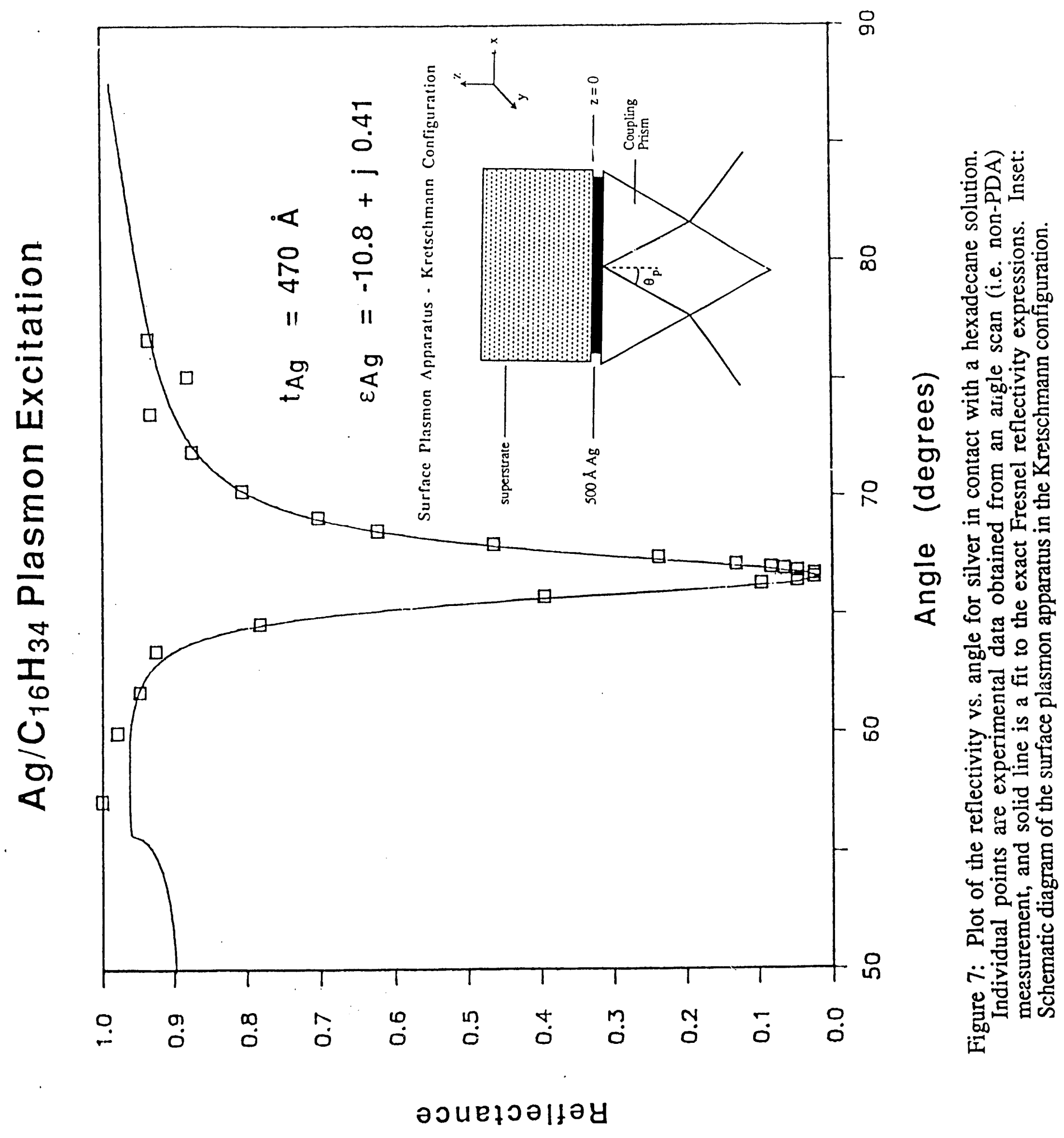


(TE) or transverse magnetic (TM) polarizations. Since the radial intensity profiles are identical for both polarizations, the TE signal, which cannot excite an SP resonance, can be used for normalization. The angular resolution external to the coupling prism is given by,

$$
\delta \theta=\frac{2 \tan ^{-1}(\mathrm{w} / 2 \mathrm{~d})}{\mathrm{N}}
$$

where $\mathrm{N}$ is the number of photodiode elementr, $w$ is the vidth of the focusing lens, and $\mathrm{d}$ is the distance from the lens to the cell. In our optical train $\mathrm{N}=1024$, the focusing lens is $5 \mathrm{~cm}$ wide and $\mathrm{d}=25 \mathrm{~cm}$, resulting in an angular resolvtion of $\delta \theta=0.01^{\circ}$. The internal angular resolution varies depending on the excitation geometry but is typically $\delta \phi=0.003^{\circ}$. The related accuracy for film thickness measurements will depend on the dielectric constants of all the materials involved in exciting the SP, but it is typically of the order of $0.5 \AA$.

The extent of the displacement reactions was followed by modeling the system as having four layers: prism/Ag/adsorbate/solvent. Initially for each sample the thickness and optical dielectric function of the silver film were determined by fitting the reflectivity obtained with neat solvent in the overlayer to the Fresnel equations. Then these Ag parameters were used for the remainder of the kinetic run. Next acetic acid was preadsorbed to produce a well-defined initial state. After the system had equilit, ated the long-chain aisid of interest was injected as a plug into the cell and the kinetic assay begun. In modeling the of ical properties of the adsorbate layer we assumed a constant refractive inaex characteristic of the bulk, and varied the thickness in our fits. Thickness values were then normalized to the thickness of a close-packed well-ordered monolayer to obtain an effective surface coverage, $\Gamma$, which was used for all comparisons among n-alkanoic acids of different chain ivingths.

Surface Plasmon Results. First it was necessary to determine the appropriate concentration range over which experiments might be performed. A minimum concentration of $0.1 \mathrm{mM}$ was selected to limit the change in bulk solution concentration upon full monolayer formation to $\leq 1 \%$. The upper concentration limit was determined by the reverse cmc. The reported reverse cmc of stearic acid in nonpolar organic solvents is typically around $1.0 \mathrm{mM}$ [29]. To measure the reverse $\mathrm{cmc}$, the surface tension of a cyclohexane solution was measured as stearic acid was added. The reverse cmc of stearic acid in cyclohexane was found to be $0.9 \pm 0.1 \mathrm{mM}$. Reverse cmc values for the other alkanoic acids in cyclohexane were

\begin{tabular}{lcc} 
Acid & Reverse CMC & Carbon Number \\
\hline Myristic Acid & $2.7 \times 10^{-3} \mathrm{M}$ & 14 \\
Palmitic Acid & $1.5 \times 10^{-3} \mathrm{M}$ & 16 \\
Stearic Acid & $0.9 \times 10^{-3} \mathrm{M}$ & 18 \\
Arachidic Acid & $0.5 \times 10^{-3} \mathrm{M}$ & 20
\end{tabular}


As expected the reverse cmc decreases with increasing chain length. Next, a concentration study was carried out for stearic acid displacement. The short-time results clearly indicated several features about these measurements and this chemical system. First excellent results can be obtained from the SP measurements even at early times. The first data point was taken at $30 \mathrm{sec}$ after injection of the displacing acid, and each data point consists of the average of 10 separate 100 msec long observations. Second, an initial reaction rate is observed which increases monotonic ily with concentration, as expected. Third, above the reverse cmc a large fraction of stearic acid molecules are aggregated in micelles, and are unable to participate in surface displacement reactions.

A comparative study of the kinetic behavior of the homologous series from myristic to arachidic acid was performed next. The chain length range was limited on the short end by lauric acid, which has been shown to form multilayers, and on the long end by arachidic acid, which has a reverse cmc of $0.5 \pm 0.1 \mathrm{mM}$. The most striking feature of the early time data is the reverse dependence of initial rate on chain length up to stearic acid, with stearic and arachidic acid being nearly indistinguishable at early times. There are two major factors which could bear upon the initial rates, desolvation and lateral interactions. From the reverse $\mathrm{cmc}$ data we expect that solventsolute interactions are more favorable for shorter chains, which is consistent with the initial rate data showing faster displacement for longer chain lengths. However, lateral interactions would also tend to favor the formation of adlayers of longer vs. shorter chains, since the van der Waals interactions can add up over more methylene units. At early times, however, it seems unlikely that displacing molecules will be sufficiertly near one another on average for this latter interaction to have a significant effect.

A model, which has been used extensively in the older literature, to describe irreversible chemisorption is based on the Elovich equation [30-33]. In this model the rate limiting step for adsorption is an energy barrier to the chemisorbed state, and the barrier height changes as a function of surface coverage, i.e. $\mathrm{E}_{\mathrm{ads}}(\Gamma)=\mathrm{E}_{\mathrm{ads}}^{\mathrm{o}}+\alpha \Gamma$ where $\mathrm{E}_{\mathrm{ads}}^{\mathrm{o}}$ is the initial adsorption energy

and $\alpha$ is a proportionality constant. Another assumption of this model is that the energy barrier for adsorption is much smaller than for desorption, and therefore desorption is considered negligible. The rate of adsorption is given by,

$$
\frac{\mathrm{d} \Gamma}{\mathrm{dt}}=\mathrm{k}_{\mathrm{a}} \exp \left(\frac{-\left(\mathrm{E}_{\mathrm{ads}}^{\mathrm{o}}+\alpha \Gamma\right)}{\mathrm{RT}}\right) \mathrm{C}_{\mathrm{o}}
$$

where $R$ is the gas constant, $T$ is the temperature, and $C_{0}$ is the bulk solution concentration of the adsorbate. An analytical solution exists for this rate law and is given by,

$$
\Gamma(t)=K_{1} \ln \left[1+k_{a} K_{1} C_{0} \exp \left(\frac{-E_{\text {ads }}^{0}}{R T}\right)\right]
$$


where $K_{1}$ is $R T / \alpha$. It is possible to solve for the proportionality constant and, if the energy barrier is known, the rate constant. Typically the Elovich equation has been applied successfully to those cases in which surface heterogeneity exists, as, for example, when there is a distribution of adsorption sites of varying potential energy barriers. In this situation the lower barrier sites are occupied initially, and higher barriers are mounted only later and and at significantly reduced rates. Unfortunately, although this type of model has been quite successful in describing the phenomenological kinetics of adsorption in many systems, its ability to yield mechanistic insight is limited. This is not surprising considering that the model results from explicit consideration of surface heterogeneity, a trait which can have any number of causes.

Fitting the adsorption kinetics to various kinetic theories shows that the goodness of fit, as measured by $\chi^{2}$, for the Elovich equation is far superior to competing models. Figure 8 shows a comparison of the Langmuir and Elovich fits to kinetic data for $0.5 \mathrm{mM}$ myristic acid. Although both equations produce acceptable fits at short times, only the Elovich equation is able to produce an acceptable fit over the entire time sequence of the displacement reaction. Since the Elovich model is predicated on a strong chemisorptive step, these data argue strongly for chemisorption. Of course, this is just what would be expected for a carboxylic acid group at a silver surface [34,35]. Data from the fits to each of the alkanoic acids at $0.5 \mathrm{mM}$ are shown below.

$\begin{array}{llll}\text { Solution } & \text { Concentration } & \alpha\left(\times 10^{-11}\right) \mathrm{erg} / \mathrm{mole} & \mathrm{k}_{\mathrm{a}} \mathrm{e}^{(-\mathrm{E} / \mathrm{RT})}(\mathrm{x} \mathrm{106}) \mathrm{min}^{-1} \mathrm{~mole}^{-1} \\ \text { Myristic Acid } & 0.5 \mathrm{mM} & 2.21 \pm 0.04 & 0.018 \pm 0.002 \\ \text { Palmitic Acid } & 0.5 \mathrm{mM} & 3.02 \pm 0.03 & 0.9 \pm 0.1 \\ \text { Stearic Acid } & 0.5 \mathrm{mM} & 3.81 \pm 0.04 & 110 \pm 10 \\ \text { Arachidic Acid } & 0.5 \mathrm{mM} & 3.7 \pm 0.1 & 40 \pm 10\end{array}$

Both the $\alpha$ parameter, which specifies the size of the perturbation to the energy barrier, and $\mathrm{k}_{\mathrm{a}} \mathrm{e}^{(\mathrm{E} / \mathrm{RT})}$ increase with increasing chain lengths up to stearic acid, and stearic acid and arachidic acid behave similarly; i.e. just the behavior expected from the early time data. The increase in $\alpha$ seems reasonable, since from steric considerations larger chain lengths are expected to physically block larger numbers of neighboring sites. This physical interpretation is consistent with the modest increase $(72 \%)$ in this parameter, since for an immobile chain interacting with the surface the blocked area would be expected to increase from ca. $82 \AA^{2}$ for myristic acid to $124 \AA^{2}$ for arachidic acid, a $50 \%$ increase. In performing this estimate it is assumed that all but two of the carbons in the chain can block surface sites. The $k_{a} e^{(E / R T)}$ rate parameter increases much more dramatically with increasing chain length, ca. 3 - 4 orders of magnitude over the range of chain lengths investigated. Finally it should be noted that variable temperature experiments were not performed due to the nature of the model used, in which the energy barrier changes with increasing surface coverage. A comparison of the adsorption of all acids over the full time scale of the experiment shows that the Elovich model is appropriate for these concentrations and chain lengths over the full adsorption process. 


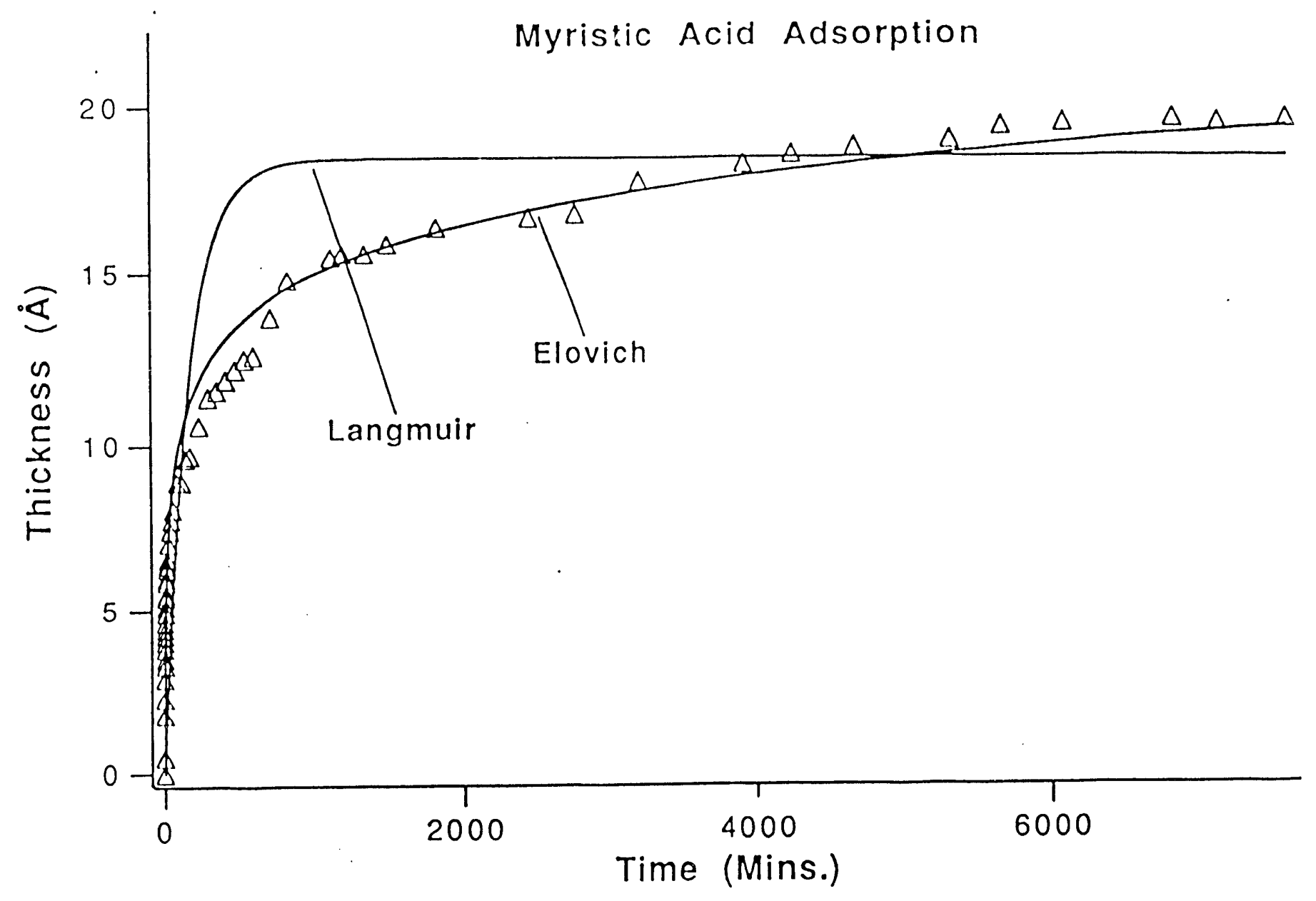

Figure 8: Comparison of Langmuir and Elovich equations for fitting kinetic data for $0.5 \mathrm{mM}$ myristic acid displacing acetic acid. The Elovich fit parameters for myristic acid are tabulated in the text. The Langmuir fit is only illustrative, since the fit is inferior to that obtained from the Elovich equation. 
Surface Raman Scattering Results. To address the question of surface rearrangement following displacement the surface Raman spectra of protonated long-chain acids displacing deuterated acetic acid in deuterated cyclohexane on $\mathrm{Ag}$ island films were studied during the evolution of the monolayer structure. Since the islands themselves are large compared to the molecular size scale (ca. $10^{2}$ molecular diameters), it is realistic to compare the results from these structure to the surface plasmon results obtained on continuous evaporated films discussed above. It is also important to realize that these real-time surface Raman scattering measurements are made possible by the ability of our CCD-based Raman spectroscopy system to acquire spectral data quickly (typically $200 \mathrm{sec}$ integrations) over the course of a surface displacement reaction. The kind of spectroscopic data to be discussed has simply not been available before in a real-time sense for surface chemical processes. The SERS spectrum of cyclohexane- $\mathrm{d}_{12}$ prior to injection of acetic acid- $\mathrm{d}_{4}$ shows only very weak solvent bands indicating that contaminant molecules are on the surface preventing enhancement of the solvent. Upon introduction of acetic acid a solvent band at $1084 \mathrm{~cm}^{-1}$ appears indicating that cyclohexane has closer access to the surface during this phase of the reaction sequence. In general this band became progressively weaker for longer chain length adsorbates. The surface Raman spectrum of a nearly complete stearic acid layer (Figure 9) indicates major bands at 1617 and $1524 \mathrm{~cm}^{-1}$ due to asymmetric carboxylate stretches [36-38]. The presence of these two bands and the lack of a band around $1700 \mathrm{~cm}^{-1}$ indicate that there is no measurable amount of free acid at the surface and that the carboxylate group is the dominant chemisorbed species both for acetic acid and the longer chain alkanoic species. The symmetric carboxylate stretch occurs at $1400 \mathrm{~cm}^{-1}$ and is the dominant peak in the spectrum. Together these three bands provide an excellent handle on the structural rearrangement possibilities in these mixed monolayer systems.

A detailed plot of the ratio of the intensity of asymmetric to symmetric carboxylate stretching vibrations for myristic acid is shown in the inset of Figure 9. By examining only ratios of bands we avoid any ambiguity associated with dipolar coupling and nonlinear surface coverage dependence in SERS. The ratio decreases in a monotonic fashion over the first 20 - 30 minutes, then reverses itself and eventually reaches a larger ratio than had been present with the preadsorbed acetic acid. The cyclohexane- $\mathrm{d}_{12}$ band at $1084 \mathrm{~cm}^{-1}$ showed a continual decrease in intensity until after 8 hours it was $24 \%$ of its original value. The decrease in the intensity is consistent with the exclusion of a greater amount of solvent from the near-surface region by the longer chain length displacing acid (vide supra). The $\mathrm{v}\left(\mathrm{CD}_{2}\right)$ bands show a similar decrease with the final intensity of all three bands being $28 \%$ of the original intensity. The correlation between the decrease in these bands and the $1084 \mathrm{~cm}^{-1}$ band supports the hypothesis that the bands are solvent bands and that as acetic acid is displaced by longer chain length alkanoic acids, solvent molecules are excluded from the vicinity of the interface, and are not enhanced as strongly in the SERS spectrum.

The only logically consistent explanation for the observed behavior, which is consistent with all available data, involves changes in the relative Raman intensities of the symmetric and asymmetric carboxylate stretches due to structural reorganization in the head group region which accompany rearrangements of the alkyl chain tails. At the beginning of the displacement reaction all of the intensity in the carboxylate stretches must come from the preadsorbed acetic acid 


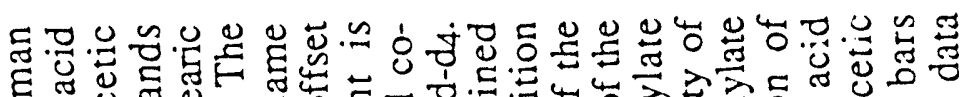

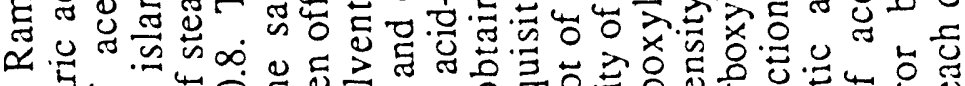

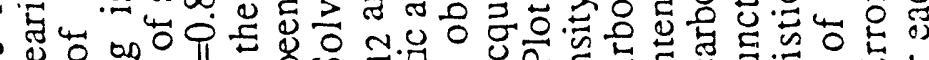

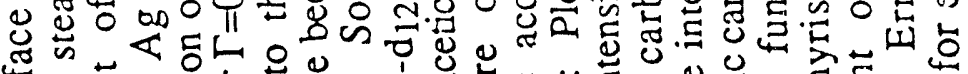

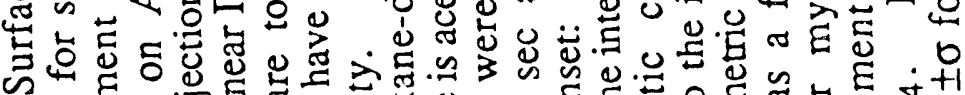
के

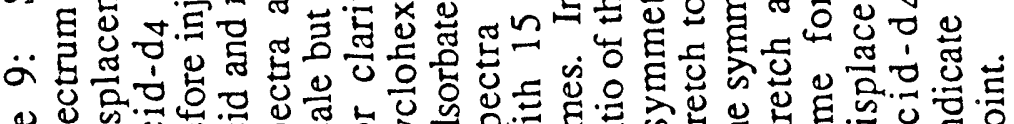

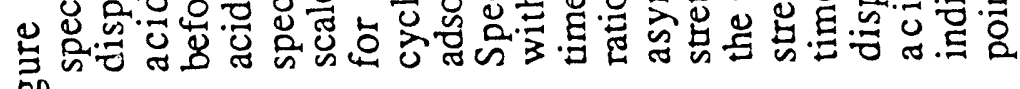
in

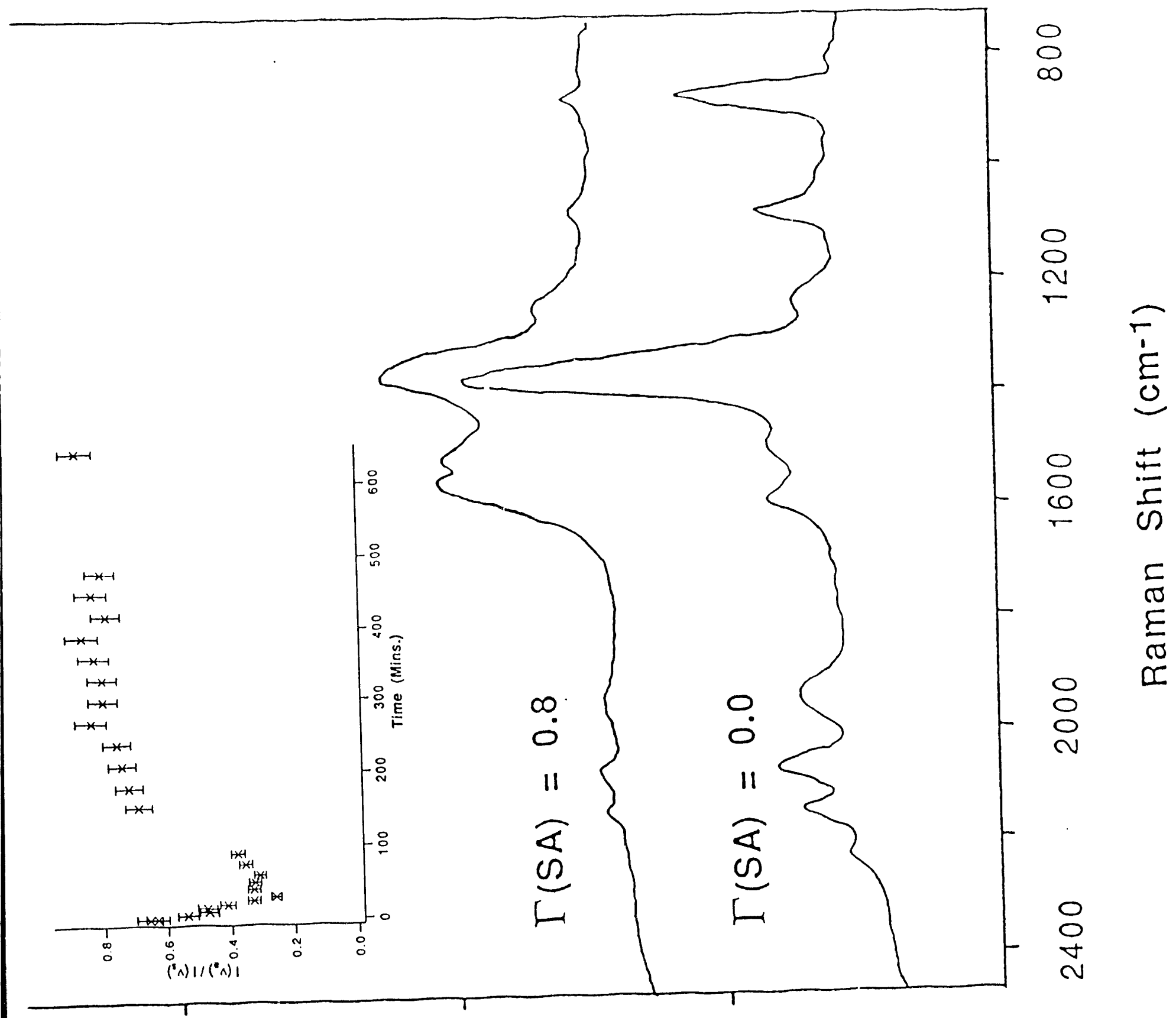


molecules. Then as longer cha:it molecules displace them, the scattering becomes a mix of contributions from both species. Finally after the knee region of the kinetic plots, i.e. at ca. 100 min., the surface is more than $50 \%$ covered with long chain molecules, and this is roughly the position at which the decreasing carboxylate stretch ratio begins to reverse itself.

These observations can be interpreted by first noting that vibrations which transform like the totally symmetric representation of the local point group will be most strongly enhanced on $\mathrm{Ag}$ island structures, since the $\alpha_{x x}$ and $\alpha_{y y}$ scattering tensor components transform like this representation. This explains the dominance of the symmetric carboxylate stretching vibration at all times. It is reasonable to expect that as the initial long chain molecules come to be bound at the surface they can bind with a variety of head and tail orientations. Then as the surface coverage become large enough that van der Waals interactions among tail groups becomes significant, order is induced in the tails, and by their covalent attachment, in the head groups as well. It is also interesting to note that the ratio of carboxylate stretches obtained from a predominantly long chain surface is roughly equivalent to the initial ratio when only acetic acid was adsorbed. This is probably due to similar head-group orientations in the two full coverage cases. This is an intuitively appealing explanation, and the only one which is substantiated by all of the experimental evidence. In order to achieve coverages approaching a rull monolayer the alkyl tails have to orient away from the surface, and interact with one another in a more or less ordered fashion. It is reasonable to expect that this is accompanied by reorientation of the head groups. Just such a reorientation was found by DuBois and coworkers in examining the adsorption of formic acid on Cu surfaces in ultrahigh vacuum $[39,40]$. Using the same two vibrations examined in the present work, they found that at low coverages the carboxylate moieties adopted a symmetric orientation perpendicular to the surface, which permitted the tails to be more nearly parallel to the surface. At higher coverages a canted carboxylate orientation was adopted, which permitted a more perpendicular arrangement of the hydrocarbon tails.

Thus, the picture which emerges involves the initial replacement of an ordered acetic acid layer by a more disordered set of stearic acid molecules initially. During this phase of the displacemeat the relative intensity of the asymmetric vibration decreases, because newly bound long-chain molecules can adopt a symmetrically bound configuration in which the $\mathrm{v}_{\mathrm{s}}\left(\mathrm{CO}_{2}^{-}\right)^{-}$is enhanced more strongly. Then as $\Gamma$ exceeds some critical ratio, which varies with chain length, the surface becomes more crowded with respect to the alkyl tails, and the head groups reorient to accommodate the tail changes. In this phase the head groups preferentially adopt a canted orientation, the $\mathrm{v}_{\mathrm{a}}\left(\mathrm{CO}_{2}^{-}\right)$vibration becomes relatively stronger, and the intensity ratio becomes larger.

Conclusions. The combination of these surface plasmon and surface Raman scattering experiments has yielded two important results. First new insights into the mechanistic and structural details of the self-assembly of an important class of model compounds, the n-alkanoic acids, have been gained. Indeed these mechanistic details, which are dominated by the effects of the alkyl tails and the van der Waals interactions among them, hold the promise of being general 
for other classes of long-chain adsorbates. The second important feature involves thic urderstanding of how to couple information from surface plasmon and surface spectroscopic experiments ،o the study of condensed-phase interfaces. The surface plasmon experiment has proven to be a highly precise and sensitive way to follow the evolution of surface coverage at solid-liquid interfaces, while the application of surface Raman spectroscopy in real-time has opened new vistas of understanding in the structural chemistry of reactive monolayer systems.

\section{Surface S rating Eabrication}

In most experiments performed with organic film waveguides simple prisms may be used to couple radiation into waveguide eigenmodes. However for precise determination of eigenmode propagation constants in solution, prisms are not satisfactory. This is because the entire apparatus must be immersed in the solution of interest. Gratings provide an efficient and robust alternative to the use of prisms for coupling. Initially experiments were performed with ca. $0.5 \mu \mathrm{m}$ spacing gratings which were fabricated using holographic lithography and reactive ion etching by Prof. G. Stegeman, Univ. of Arizona. This spacing is satisfactory to obtain coupling in the first diffraction order for all the organic films being investigated in these experiments. Over time these i:iitial gratings samples were broken primarily because of stresses associated with mounting the samples in the liquid cell, so $t$ was decided to develop our own capability to fabricate these structures. We have now successfully demonstrated this capability.

The procedure follows closely that of Stegeman [41]. The process begins with holographic exposure of AZ1350J-SF in a Lloyd's mirror arrangement at $457.9 \mathrm{~nm}$. In this arrangement grating spacing is adjusted simply by changing the angle of incidence (and therefore the angle at which the two coherent beams interfere). Development of the resist transfers the pattern into the resist. Initial experiments focused on the use of chemical (buffered HF at various concentrations and ionic strengths) etches to transfer the pattern into the silica substrates. These experiments were all plagued by photoresist lift-off during the etching step. Infrequently a resist layer would stick long enough to achieve a significant transfer. The best diffraction efficiencies from gratings processed in this manner were in the range $2-4 \%$. Since this was deemed inadequate for our experiments, the final chemical etching step was replaced with a reactive ion etch in $\mathrm{CF}_{4}$. These transfers were much more efficient, yielding gratings with first order diffraction efficiencies in the range $20-25 \%$. These structures are now the substrates of choice for all liquid-interface optical waveguide experiments.

The high quality of these gratings structures is attested by a typical eigenmode distribution measurement for the heavily-moded P2VP guide shown in Figure 10. Here the mode halfwidth is of the order $0.24^{\circ}$, a factor of $2-4$ smaller than the best results obtained with prism coupling. In addition with prism coupling it would require extreme care to keep the substrate-prism gap aligned over the angular range covered by all the modes. Recovery of the optical properties for the film 


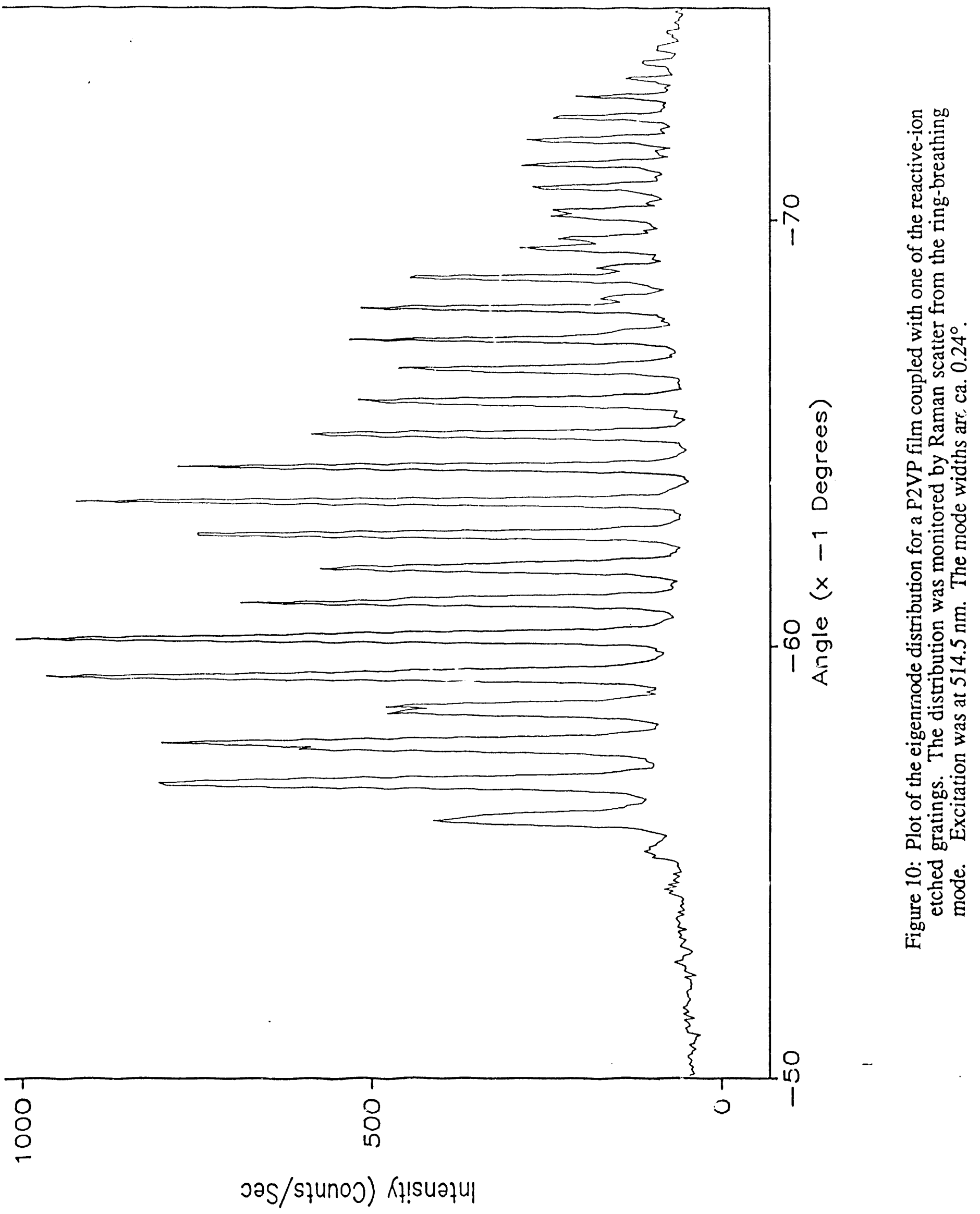


showed $\{\mathrm{n}, \mathrm{t}\}=\{1.6059 \pm 0.0001,12.696 \pm 0.055 \mu \mathrm{m}\}$, the best precision ever attained in our laboratory for an $\{n ;\}$ measurement.

\section{Publications}

The followin , manuscripts and publications have resulted from support by this DOE grant.

A.R. Siirila and P.W. Bohn, "The Kinetics of Carboxylic Acid Surface Displacement Reactions: A Coupled Surface Plasmon and Surface Raman Scattering Investigation," Langmuir, submitted.

N.F. Fell and P.W. Bohn, "In Situ Evaluation of Diffusion Mechanisms in Thin Polystyrene Films Used as Integrated Optical Structures," Appl. Spectrosc.., in press.

P.W. Bohn and D.R. Miller, "Depth Profiling in Thin Dielectric Films," Crit. Rev. Analyt. Chem., in press.

P.W. Bohn and D.J. Walls, "Challenges in the Structural Characterization of Thin Organic Films," Mikrochim. Acıa, 1991, I, 3-35.

P.W. Bohn and N.F. Fell, "In Situ Studies of Modified Organic Thin Films with Optical Guided Waves," Proc. SPIE, 909, 130-137 (1989). 


\section{References}

(1) N.L. Thomas and A.H. Windle, Polymer 21, 613 (1980).

(2) N.L. Thomas and A.H. Windle, Polymer 22, 627 (1981).

(3) N.L. Thomas and A.H. Windle, Polymer 23, 529 (1982).

(4) G.C. Sarti, Polymer 20, 827-832 (1979).

(5) R.W. Korsmeyer, S.R. Lustig, and N.A. Peppas, J. Polym. Sci. Polym. Phys. Ex . 4, 395 (1986).

(6) R.W. Korsmeyer, E. von Meerwall, and N.A. Peppas, J. Polym.Sci. Polym. Phys. Ed. 24, 409 (1986).

(7) C.-Y. Hui, K.-C. Wu, R.C. Lasky, and E.J. Kramer, J. Appl. Phys. 61, 5129 (1987).

(8) C.-Y. Hui, K.-C. Wu, R. C. Lasky, and E.J. Kramer, J. Appl. Phys. 61, 5137 (1987).

(9) L. Nicolais, E. Drioli, H.B. Hopfenburg, and D. Tidone, Polymer 18, 1137 (1977).

(10) L.A. Weisenterger and J.L. Koenig, Appl. Spectrosc. 43, 1117 (1989).

(11) J.S. Papanu, D.W. Hess, A.T. Bell, and D.S. Soane, J. Electrochem. Soc. 136, 1195 (1989).

(12) P.J. McMarr, K. Vedam, and J. Narayan, J. Appl. Phys. 59, 694 (1985).

(13) K. Vedam, S.Y. Kim, L. D'Aries, and A.H. Guenther, Opt. Lett. 12, 456 (1987).

(14) M.W. Urban and J.L. Koenig, Appl. Spectrosc. 40, 994 (1986).

(15) J.C. Donini and K.H. Michaelian, Infrared Phys. 24, 157 (1984).

(16) C.W. Magee and W.L. Harrington, Appl. Phys. Lett. 33, 193 (1978).

(17) P.J. Mills, P.F. Green, C.J. Palmstrøm, J.W. Mayer, and E.J. Kramer, Appl. Phys. Lett. 45, 957 (1984).

(18) D.R. Miller, O.H. Han, and P.W. Bohn, Appl. Spectrosc. 41, 245 (1987).

(19) D.R. Miller, O.H. Han, and P.W. Bohn, Appl. Spectrosc. 41, 249 (1987).

(20) P.W. Bohn and N.F. Fell, Proc. Soc. Photo-Opt. Instrum. Eng. 990, 130 (1988).

(21) D.R. Miller and P.W. Bohn, Anal. Chem. 60, 407 (1988).

(22) D.R. Miller and P.W. Bohn, Appl. Opt. 27, 2561 (1988).

(23) R. Ulrich and R. Torge, Appl. Opt. 12, 2901 (1973).

(24) J.D. Swalen, R. Santo, M. Tacke, and J. Fischer, IBM J. Res. Dev. 21, 168 (1977).

(25) J.D. Swalen, M. Tacke, R. Santo, and J. Fischer, Opt. Comm. 18, 387 (1976).

(26) A.S. Michaels, H.J. Bixler, and H.B. Hopfenburg, J. Appl. Polym. Sci. 12, 991 (1968).

(27) J. Sagiv,J. Gun, R. Maoz, and L. Netzer in "Surfactants in Solution," v.5; K.L. Mittal and P. Bothoral, Eds., Plenur ess, New York, 1986.

(28) K. Matsubara, S. Kawata, and S. Minami, Applied Optics 27, 1160 (1988).

(29) P.C. Heimenz,, "Principles of Colloid and Surface Chemistry," Marcel Dekker, New York, 1986, pp. 463-467. -

(30) S.Z. Roginsky and J. Zeldovich, Acta Physicochim. USSR 1, 554 (1934).

(31) G.A. Taylor and N. Thon, J. Am. Chem. Soc. 74, 4169 (1952).

(32) M.J.D. Low, Chem. Rev. 60, 267 (1960).

(33) I.S. McClintock, Nature 216, 1204 (1967).

(34) C.O. Timmons and W.A. Zisman, J. Phys. Chem. 69, 984 (1965).

(35) W.C. Bigelow, D.L. Pickett, and W.A. Zisman, J. Coll. Interf. Sci. 1, 513 (1946). 
(36) D.L. Allara and R.G. Nuzzo,Langmuir 1, 52 (1985).

(37) Y. Kim and K. Machida, Spectrochim. Acta 42A, 881 (1986).

(38) R.P. Groff, J. Catal. 79, 259 (1983).

(39) L.H. DuBois, T.H. Ellis, B.R. Zegarski, and S.D. Kevan, Surf. Sci. 172, 385 (1986).

(40) L.H. Dubois, B.R. Zegarski, and R.G. Nuzzo, Langmuir 2, 412 (1986).

(41) X. Mai, R. Moshrefzadeh, U.J. Gibson, G.I. Stegeman, and C.T. Seaton, Appl. Opt. 24, 3155 (1985). 

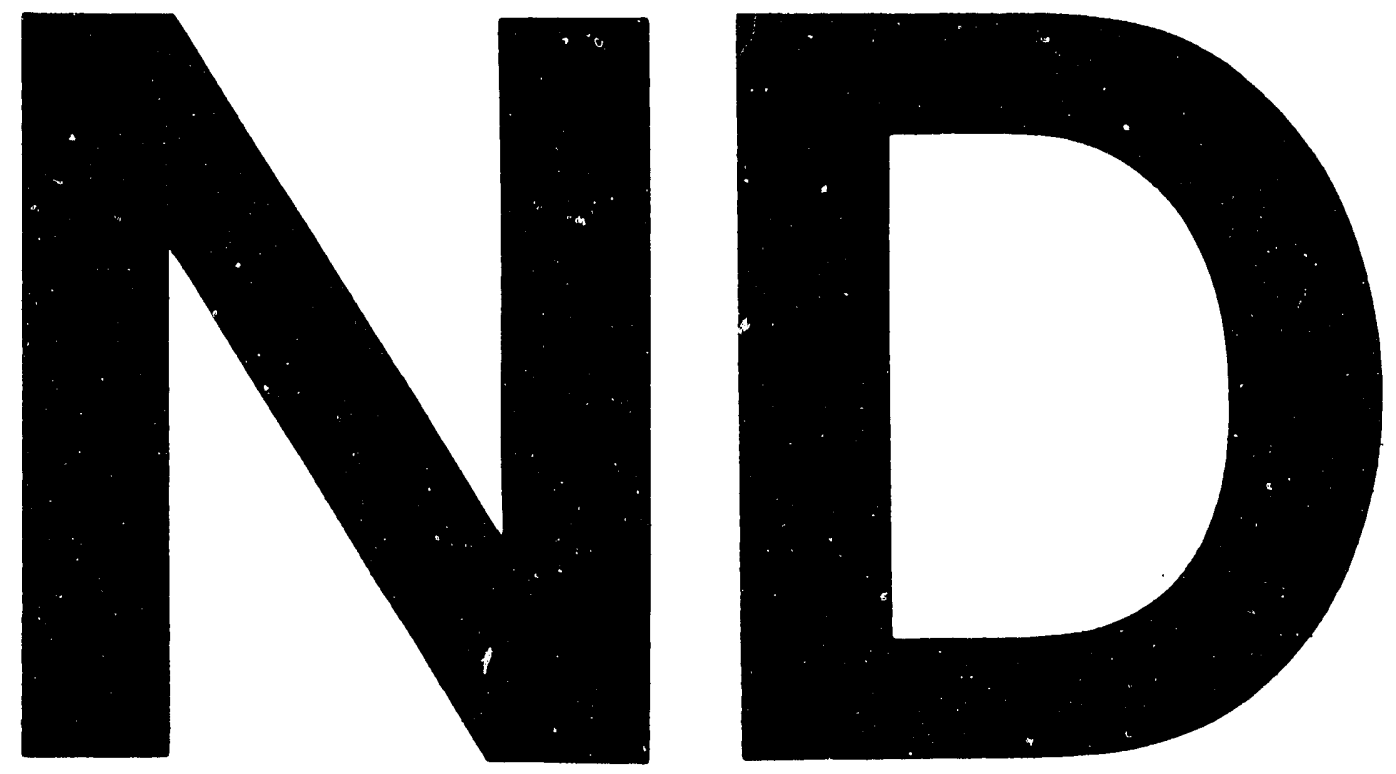

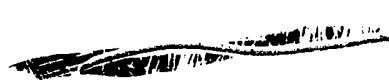
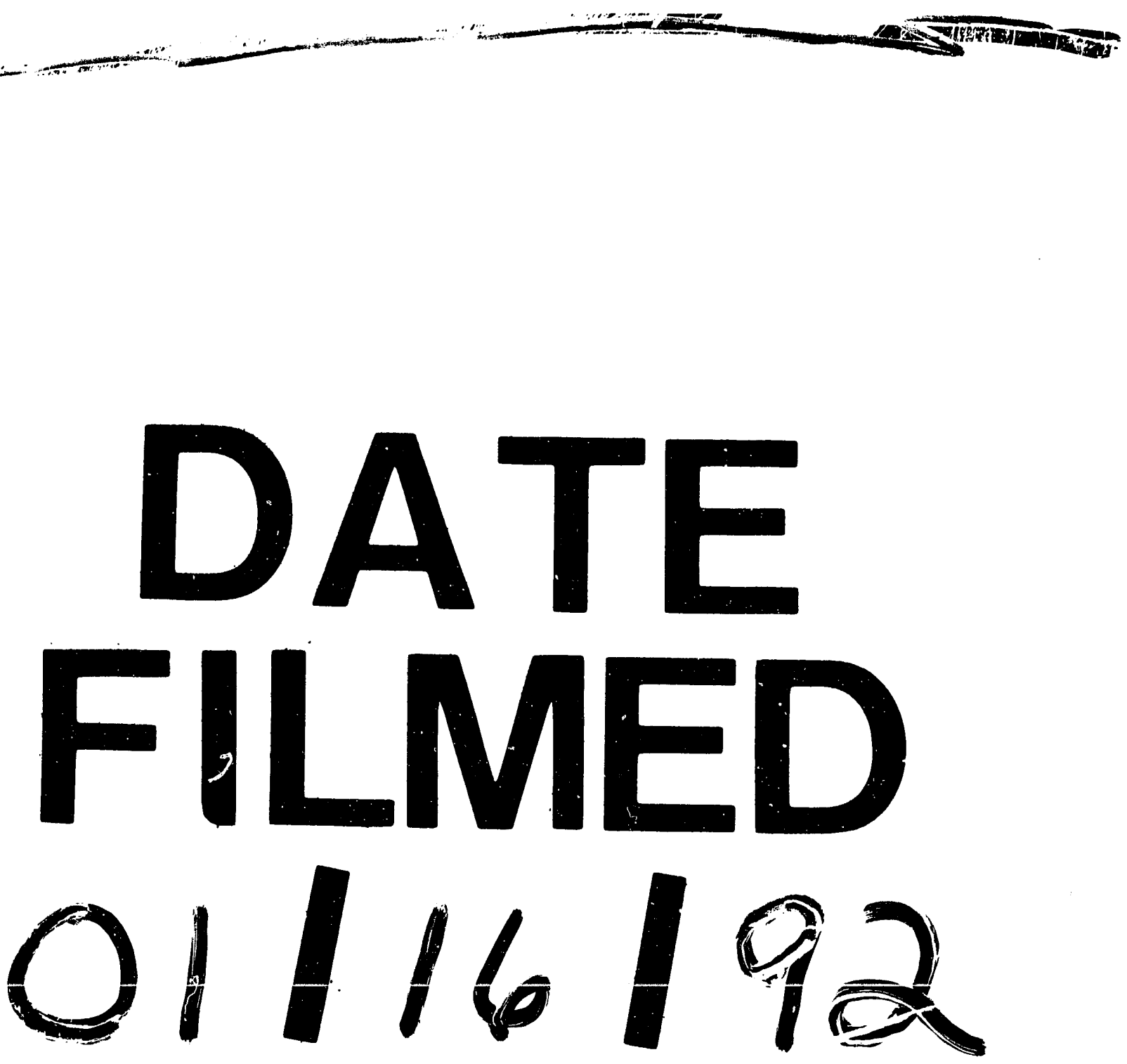
